\title{
Robust Stability for a Class of Uncertain Singular Time-Delays Systems
}

\author{
Mingliang Li, Yali Dong*, Jinying Liu, Chaoyue Zhao \\ * School of Science, Tianjin Polytechnic University, \\ Tianjin 300160, China \\ *Corresponding authors Email: author@yahoo.com
}

\begin{abstract}
This note provides new stability criteria for a class of uncertain singular systems with multiple-state delays. By introducing the state transformation, the study of the robust stability for original systems is changed into this study for the equivalent systems. Based on the Lyapunov-Krasovskii functional combination with LMI techniques, a delay-dependent robust stability criterion for the nominal systems of a class of uncertain singular systems is established, which ensures the nominal systems are asymptotically stable. Furthermore, the delay-dependent robust stability criterion for a class of uncertain singular systems with multiple-state delays is presented, which ensures the class of uncertain singular systems is asymptotically stable. Finally, two numerical examples are given to illustrate the effectiveness of the obtained results.
\end{abstract}

Keywords: robust stability, time-delay, linear matrix inequality (LMIs), Lyapunov-Krasovskii functional, delaydependent

\section{Introduction}

Time delays are frequently encountered in many dynamical systems, such as manufacturing systems, economic systems, biological systems, networked systems, etc. They are generally regarded as a main source of instability and poor performance in such systems. One of the main purposes to analysize linear differential systems is to analyze the stability of the systems, especially the certain and uncertain linear differential control systems with time delay, which is important not only in theory but also in practice and arouses a lot of interests. Some results are obtained [1-5]. A delay-dependent criterion for determining the stability of systems with time-varying delays is obtained by combing a new approach for linear time delay systems based on a descriptor representation with a result on bounding of cross products of vectors in [1]. In [4], the system with single state delay and uncertainty is considered, and non-conservative results are obtained by using new Lyapunov-krasovskii functionals. The stability of regular systems with multiplestate delay and uncertainty is considered in [6], and based on Lyapunov-Krasovskii functionals combined with LMI techniques. Thus delay-dependent robust stability criteria are given.

It should be pointed out that the problem for singular systems is more complicated than that for regular systems, and singular systems better describe physical systems than regular ones, so the singular systems have been extensively studied in the past years [7-10].

Study of singular systems with time delay is of recurring interest $[8,10]$. The robust stability and robust stabilization of uncertain singular systems with single state delay are considered in [8]. The delaydependent robust stability criteria for two classes of singular time-delay systems with norm-bounded uncertainties are proposed in [11], which ensure that the systems are regular, impulse free and asymptotically stable for all admissible uncertainties. A great num- 
ber of results based on the theory of regular systems have been extended to the area of singular systems $[9,10]$. Generally speaking, the existing results of stability and stabilization for singular delay systems can be classified into two types: delay-independent conditions and delay-dependent conditions, and the delay- independent case is more conservative than the delay-dependent case, especially when the time delay is comparatively small. The delay-independent case has been extensively studied $[13,14]$. Recently, the problem of delay-dependent robust stability for uncertain discrete singular time-delay systems has been considered. In [15], the delay-dependent robust stabilization result is proposed by transforming the system into a standard state-space system.

This paper considers the robust stability of a class of uncertain singular systems with multiple-state delays. We obtain the transforming uncertain singular systems which are called the equivalent system with multiple-state delays via state transformation matrix, so the research of the original system is changed into the research of equivalent system. First, this brief constructs Lyapunov-Krasovskii functionals, which are applied to the equivalent nominal systems and equivalent systems respectively. Then we present the delaydependent stability criteria, which ensure that the equivalent systems are asymptotical stable. It ensures that the original systems are asymptotically stable at the same time.

Notations: $R$ denotes the set of real numbers, $R^{n}$ denotes the $n$-dimensional Euclidean space over the real and $R^{n \times m}$ denotes the set of all $n \times m$ real matrices. For a real symmetric matrix $X$, the notation $X \geq 0(X>0)$ means that the matrix $X$ is positivesemidefinite (positive-definite), and $\lambda_{\min }(X)\left(\lambda_{\max }(X)\right)$ denotes the minimum (maximum) eigenvalues of $X$. $C_{n, \tau}:=C\left([-\tau, 0], R^{n}\right)$ denotes the Banach space of continuous vector functions mapping the interval $[-\tau, 0]$ into $R^{n}, x_{t}:=x(t+\theta), \theta \in[-\tau, 0], t \geq 0$ denotes the function family defined on $[-\tau, 0]$ which is generated by $n$-dimensional real vector valued continuous function $x(t) . t \in[-\tau, \infty] .\|$.$\| refers to the Euclidean vector$ norm or spectral matrix norm,

$$
\|\phi\|:=\sup _{-\tau \leq t \leq 0}\|\phi(t)\|
$$

stands for the norm of a function $\phi(t) \in C_{n, \tau}$. The symbol * will be used in some matrix expressions to induce a symmetric structure, for example,

$$
\left[\begin{array}{ll}
X & Y \\
* & Z
\end{array}\right]=\left[\begin{array}{cc}
X & Y \\
Y^{T} & Z
\end{array}\right]
$$

\section{System Description}

Consider the following uncertain time-delay singular systems described by

$\left\{\begin{array}{l}E \dot{X}(t)=\left(A_{0}+\triangle A_{0}(x, t)\right) x(t)+\sum_{k}^{i=1}\left(A_{i}+\triangle A_{i}(x, t)\right) x\left(t-h_{i}\right) \\ x(t)=\phi(t) \forall t \in[-\mathrm{h}, 0]\end{array}\right.$

where $x(t) \in R^{n}$ is the state vector, $A_{j}, j=0,1, \ldots, k$, are known constant matrices with appropriate dimension, $\triangle A_{j}(x, t), j=0,1, \ldots k$ are matrix functions representing the uncertainties in the matrices $A_{j}, j=0,1, \ldots k$. $E$ is a singular matrix. Without the lose of generality, we can assume that

$$
\begin{aligned}
& E=\left[\begin{array}{cc}
I_{r} & 0 \\
0 & 0
\end{array}\right] \\
& A_{i}=\left[\begin{array}{ll}
A_{i 11} & A_{i 12} \\
A_{i 21} & A_{i 22}
\end{array}\right], A_{i 11} \in R^{r \times r}, i=0,1, \ldots k, \\
& x(t)=\left[\begin{array}{l}
x_{1}(t) \\
x_{2}(t)
\end{array}\right], \phi(t)=\left[\begin{array}{l}
\phi_{1}(t) \\
\phi_{2}(t)
\end{array}\right], \\
& x_{1}(t), \phi_{1}(t) \in R^{r \times r}, \\
& \triangle A_{j}(x, t)=D_{j} F_{j}(x, t) E_{j}, j=0,1, \ldots, k
\end{aligned}
$$

where $F_{j}(x, t) \in R^{k_{j} \times g_{j}}$ are unknown real time-varying matrices bounded by

$$
F_{j}^{T}(x, t) F_{j}(x, t) \leq I, \forall t, j=0,1, \ldots k
$$

$D_{j}$ and $E_{j}$ are known real constant matrices, $h_{i}, i=$ $0,1, \ldots, k$, are the unknown constant delay terms, but bounded $0 \leq h_{i} \leq h . \phi(t)$ is a smooth vector-valued initial function in $-h \leq t \leq 0$.

In the following, for simplicity, we denote $\triangle A_{j}(x, t)=$ $\triangle A_{j}(t)$.

The aim of this paper is to develop delay-dependent conditions for robust stability of the uncertain timedelay system (1).

The following lemma is needed in the proof of this paper.

Lemma 1[12] Let $D, E$ and $F$ be real matrices of appropriate dimensions with $\|F\| \leq 1$, then for any scalar $\varepsilon>0$, we have the following inequality:

$$
D F E+E^{T} F^{T} D^{T} \leq \varepsilon^{-1} D D^{T}+\varepsilon E^{T} E
$$

Lemma 2[16] (Schur complement) Given constant symmetric matrices $S_{1}, S_{2}, S_{3}$, where $S_{1}=S_{1}^{T}, 0<S_{2}=$ $S_{2}^{T}$, then $S_{1}+S_{3} S_{2}^{-1} S_{3}<0$ if and only if

$$
\left[\begin{array}{cc}
S_{1} & S_{3}^{T} \\
S_{3} & -S_{2}
\end{array}\right]<0, \text { or }\left[\begin{array}{cc}
-S_{2} & S_{3} \\
S_{3}^{T} & S_{1}
\end{array}\right]<0
$$




\section{Main Results}

Let

$$
z(t)=e^{\alpha t} x(t), t>0
$$

where $\alpha>0$ is stability degree. Differentiating $z(t)$ with respect to $t$, we have

$$
\dot{z}(t)=\alpha e^{\alpha t} x(t)+e^{\alpha t} \dot{x}(t)=\alpha z(t)+e^{\alpha t} \dot{x}(t)
$$

Then, from (1), (5) and (6), we have

$$
\begin{aligned}
E \dot{z}(t)= & \alpha E z(t)+e^{\alpha t} E \dot{x}(t) \\
= & \left(\alpha E+A_{0}+\triangle A_{0}\right) z(t) \\
& +\sum_{i=1}^{k}\left(A_{i}+\triangle A_{i}\right) e^{\alpha h_{i}} z\left(t-h_{i}\right) \\
Z(t)= & e^{\alpha t} \phi(t), \forall t \in[-h, 0]
\end{aligned}
$$

So the research for the system (1) is changed into the research for the system (7).

The nominal singular delay system of (1) can be written as

$$
\left\{\begin{array}{l}
E \dot{X}(t)=A_{0} x(t)+\sum_{k}^{i=1} A_{i} x\left(t-h_{i}\right) \\
x(t)=\phi(t) \forall t \in[-h, 0]
\end{array}\right.
$$

Throughout this paper we shall use the following concept of robust stability for uncertain systems (7).

Definition 1[7] The singular system (7) is said to be robust stable if for any $\varepsilon>0$, there exist a $\delta(\varepsilon)>0$ such that for all continuous $e^{\alpha t} \phi(t)$, with $e^{\alpha t} \phi(t)$ satisfying the solution to (7) satisfying $\sup _{-\tau \leq r \leq 0}\left\|e^{\alpha t} \phi(t)\right\| \leq$ $\delta(\varepsilon)$, the solution $z(t)$ to (7) satisfying $\|z(t)\| \leq \varepsilon$ for all $t \geq 0$ The solution of (7) is said to be robust asymptotically stable if it is stable and furthermore $z(t) \rightarrow 0$, when $t \rightarrow \infty$.

Remark 1 Because $x(t)=e^{-\alpha t} z(t)$, where $\alpha>0$, the asymptotical stability of the system (7) ensures the asymptotically stability of the system (1), and we transform the research for the system (1) into the research for the system (7).

First of all, we will investigate the nominal system stability of system (7).

The nominal singular delay system of (7) can be written as

$$
\begin{aligned}
E \dot{X}(t) & =\alpha E z(r)+e^{\alpha t}\left(A_{0} x(t)+\sum_{i=1}^{k} A_{o} x\left(t-h_{i}\right)\right) \\
& =\left(A_{0}+\alpha E\right) z(t)+\sum_{i=1}^{k} A_{i} e^{\alpha h_{i}} z\left(t-h_{i}\right) \\
z(t) & =e^{\alpha t} \phi(t), \forall t \in[-h, 0]
\end{aligned}
$$

Remark 2 If $A_{022}$ is non-singular, then the system (9) is regular and impulse free [17]. In this case, the compatible initial condition is

$$
\begin{aligned}
0= & A_{021} \phi_{1}(0)+A_{022} \phi_{2}(0)+\sum_{i=1}^{k} e^{\alpha h_{i}} A_{i 21} z_{1}\left(t-h_{i}\right) \\
& +\sum_{i=1}^{k} e^{\alpha h_{i}} A_{i 22} z_{2}\left(t-h_{i}\right)
\end{aligned}
$$

Define the difference operator $D: C_{n-r, r} \rightarrow R^{n-r}$

$$
D\left(z_{2 t}\right)=z_{2}(t)+\sum_{i=1}^{k} e^{\alpha h_{i}} A_{022}^{-1} A_{i 22} z_{2}\left(t-h_{i}\right)
$$

That the operator is stable means that the equation $D_{z_{2 t}}=0$ is asymptotically stable.

We need the following lemma.

Lemma 3 [10] If the operator $D$ is stable and there exist positive number $\alpha, \beta, \gamma$ and a continuous functional $V: C_{n, \tau} \rightarrow R$ such that

$$
\begin{array}{r}
\alpha\left\|Z_{1}(t)\right\| \leq V\left(z_{t}\right) \leq \beta\left\|z_{t}\right\|_{c}^{2} \\
\dot{V}\left(z_{t}\right) \leq-\gamma\|z(t)\|^{2}
\end{array}
$$

and the function $\bar{V}(t)=V\left(z_{t}\right)$ is absolutely continuous for $z_{t}$ satisfying (9), then (9) is asymptotically stable.

In the following, we give the asymptotically stable condition of the system (8).

Theorem 1 Consider the singular delay system (8) with all constant delays $h_{i} \in[0, h], i=0,1, \ldots, k$. Then the system (8) is asymptotically stable if there exist $0<R \in R^{n \times n}, i=1, \ldots, k, P=\left[\begin{array}{cc}P_{1} & 0 \\ P_{2} & p_{3}\end{array}\right] \in R^{n \times n}$, with $0<P_{1} \in R^{r \times r}$, and a constant $\alpha>0$, such that the following LMI holds:

$$
\left[\begin{array}{cc}
X_{1} & M_{1} \\
M_{1}^{T} & -N_{1}
\end{array}\right]<0
$$

where

$$
\begin{aligned}
X_{1} & =P^{T} A_{0}+A_{0}^{T} P+\alpha P^{T} E+\alpha E P+\sum_{i=1}^{k} R_{i} \\
M_{1} & =\left[e^{\alpha h_{1}} P^{T} A_{1} \ldots e^{\alpha h_{k}} P^{T} A_{1}\right] \\
N_{1} & =\operatorname{diag} R_{1}, \ldots, R_{k}
\end{aligned}
$$

Proof Let $z(t)=e^{\alpha t} x(t)$ then the system (8) is transformed into the system (9). From (11), we get . (12)

$$
X_{1}<0
$$


From (2) and (12), we have

$\left[\begin{array}{cc}\Gamma_{11} & P_{1}^{T} A_{012}+P_{2}^{T} A_{022}+A_{021}^{T} P_{3}+\sum_{i=1}^{k} R_{i 12} \\ \Gamma_{21} & P_{3}^{T} A_{022}+A_{022}^{T} P_{3}+\sum_{i=1}^{k} R_{i 22}\end{array}\right]<0$

where

$$
\begin{aligned}
\Gamma_{11}= & P_{1}^{T} A_{011}+P_{2}^{T} A_{021}+A_{011}^{T} P_{2}+A_{021}^{T} P_{2}+\alpha P_{1} \\
& +\alpha P_{1}^{T}+\sum_{i=1}^{k} R_{i 11} \\
\Gamma_{21}= & A_{012}^{T} P_{1}+A_{022}^{T} P_{2}+P_{3}^{T} A_{021}+\sum_{i=1}^{k} R_{i 12}^{T}
\end{aligned}
$$

Hence, it is yielded that

$$
P_{3}^{T} A_{022}+A_{022}^{T} P_{3}+\sum_{i=1}^{k} R_{i 22}<0
$$

Noticing $\sum_{i=1}^{k} R_{i 22}>0$ we get $P_{3}^{T} A_{022}+A_{022}^{T} P_{3}<0$, which implies that $A_{022}$ is non-singular. Therefore, the system (9) is regular and impulse free [17]. According to Lemma 2 in [7], we know that the operator $D$ is stable.

Constructing the Lyapunov-Krasovskii functionals for the system (9) as follows:

$$
\begin{aligned}
V\left(z(t), z\left(t-h_{1}\right), \ldots, z\left(t-h_{k}\right)\right) & =z^{T}(t) E P z(t)+ \\
& \sum_{i=1}^{k} \int_{t-h_{i}}^{t} z^{T}(\theta) R_{i} z(\theta) d \theta
\end{aligned}
$$

we get

$$
t \geq h_{i}(i=1,2, \ldots k),\left\|z_{t}\right\|_{c}=\sup _{\theta \in[-\tau, 0]}\|z(t+\theta)\|
$$

Because

$$
\begin{aligned}
\frac{d}{d t}\left(z^{T}(t) E P z(t)\right) & =2 z_{1}^{T}(t) P_{1} \dot{z}_{1}(t) \\
& =2 z_{1}^{T}(t) P^{T}\left[\begin{array}{c}
\dot{z}_{1}(t) \\
0
\end{array}\right]
\end{aligned}
$$

the time derivative of along the trajectory of (9) is given by

$$
\begin{gathered}
\dot{V}\left(z(t), z\left(t-h_{1}\right), \ldots, z\left(t-h_{k}\right)\right)=2 z^{T}(t) P^{T}\left[\begin{array}{c}
\dot{z}_{1}(t) \\
0
\end{array}\right] \\
+\sum_{i=1}^{k}\left(z^{T}(t) R_{i} z(t)-z^{T}\left(t-h_{i}\right) R_{i} z\left(t-h_{i}\right)\right) \\
=2 z^{T}(t) P^{T}\left[\left(A_{0}+\alpha E\right) z(t)+\sum_{i=1}^{k} A_{i} e^{\alpha h_{o}} z\left(t-h_{i}\right)\right] \\
+\sum_{i=1}^{k}\left(z^{T}(t) R_{i} z(t)-z^{T}\left(t-h_{i}\right) R_{i} z\left(t-h_{i}\right)\right)
\end{gathered}
$$

$$
\begin{aligned}
= & z^{T}(t)\left(2 P^{T} A_{0}+2 \alpha P^{T} E+\sum_{i=1}^{k} R_{i}\right) z(t) \\
& +z^{T}(t)\left(2 P^{T} \sum_{i=1}^{k} A_{i} e^{\alpha h_{i}}\right) z\left(t-h_{i}\right) \\
& -\sum_{i=1}^{k} z^{T}\left(t-h_{i}\right) R_{i} z\left(t-h_{i}\right)
\end{aligned}
$$

We get

$$
\dot{V}\left(z(t), z\left(t-h_{1}\right), \ldots, z\left(t-h_{k}\right)\right)=\xi^{T} S \xi
$$

where

$$
\begin{aligned}
\xi & =\left[\dot{V}\left(z(t), z\left(t-h_{1}\right), \ldots, z\left(t-h_{k}\right)\right)\right]^{T} \\
S & =\left[\begin{array}{cc}
X_{1} & M_{1} \\
M_{1}^{T} & -N_{1}
\end{array}\right]
\end{aligned}
$$

$M_{1}, X_{1}, N_{1}$ are defined in (11)

From condition (11), we have . Thus, from the stability of operator, Lemma 3 and (13), it follows that system (9) is asymptotically stable. So the system (8) is asymptotically stable. This completes the proof.

Theorem 2 Consider the singular delay system (1) with $\triangle A_{j}(t)=D_{j} F_{j}(t) E_{j}, j=0,1, \ldots k,\|F\| \leq 1$ For all delays given $h_{i} \in[0, h], i=1,2, \ldots k$, the system (1) is robust asymptotically stable if there exist $0<R \in$ $R^{n \times n}, i=1, \ldots, k, P=\left[\begin{array}{cc}P_{1} & 0 \\ P_{2} & p_{3}\end{array}\right] \in R^{n \times n}$, with $0<$ $P_{1} \in R^{r \times r}$ and scalars $\varepsilon_{j}>0, j=1, \ldots, k$, such that the following LMI holds:

$$
\left[\begin{array}{cccc}
X_{2} & M_{1} & K & M_{3} \\
M_{1}^{T} & -N_{2} & 0 & 0 \\
K^{T} & 0 & -T & 0 \\
M_{3}^{T} & 0 & 0 & -N_{3}
\end{array}\right]<0
$$

where

$$
\begin{aligned}
& X_{2}=P^{T} A_{0}+A_{0}^{T} P+\alpha P^{T} E+\alpha E P+ \\
& K=P T D_{1} \quad+P_{i=1}^{k} R_{i}+\varepsilon_{0} E_{0}^{T} E \\
& K=P T D_{1} \quad \ldots \quad P^{T} D_{k} \\
& T=\text { diage }^{-2 \alpha h_{1}} \varepsilon_{1} I_{k_{1}} \quad \ldots \quad e^{-2 \alpha h_{k}} \varepsilon_{k} I_{k_{k}} \\
& M_{3}=P^{T} D_{0}, N_{3}=\varepsilon_{0} I_{k_{0}} \\
& M_{1}=\left[\begin{array}{lll}
e^{-2 \alpha h_{1}} P^{T} A_{1} & \ldots & e^{-2 \alpha h_{k}} P^{T} A_{k}
\end{array}\right] \\
& N_{2}=\operatorname{diag} R_{1}-\varepsilon_{1} E_{1}^{T} E_{1}, \ldots, R_{k}-\varepsilon_{k} E_{k}^{T} E_{k}
\end{aligned}
$$

Proof Using we transform (1) into the system (7).

Similar to the proof in theorem 1, we get that the operator $D$ is stable. 
Constructing the Lyapunov-Krasovskii functional for system (7) as follows:

$$
\begin{gathered}
V\left(z(t), z\left(t-h_{1}\right), \ldots, z\left(t-h_{k}\right)\right)=z^{T}(t) E P z(t)+ \\
\sum_{i=1}^{k} \int_{t-h_{i}}^{t} z^{T}(\theta) R_{i} z(\theta) d \theta \\
\dot{V}\left(z(t), z\left(t-h_{1}\right), \ldots, z\left(t-h_{k}\right)\right)=2 z^{T}(t) P^{T}\left[\begin{array}{c}
\dot{z}_{1}(t) \\
0
\end{array}\right] \\
\quad+\sum_{i=1}^{k}\left(z^{T}(t) R_{i} z(t)-z^{T}\left(t-h_{i}\right) R_{i} z\left(t-h_{i}\right)\right) \\
=2 z^{T}(t) P^{T}\left[\left(\alpha E+A_{0}+\triangle A_{0}\right) z(t)\right. \\
\left.\quad+\sum_{i=1}^{k}\left(A_{i}+\triangle A_{i}\right) e^{\alpha h_{i}} z\left(t-h_{i}\right)\right] \\
\quad+\sum_{i=1}^{k}\left(z^{T}(t) R_{i} z(t)-z^{T}\left(t-h_{i}\right) R_{i} z\left(t-h_{i}\right)\right)
\end{gathered}
$$

Hence we have

$$
\dot{V}\left(z(t), z\left(t-h_{1}\right), \ldots, z\left(t-h_{k}\right)\right)=\xi^{T}(S+\bar{S}) \xi
$$

where

$$
\begin{aligned}
& \bar{S}=\left[\begin{array}{cc}
\Gamma & M_{2} \\
M_{2}^{t} & 0
\end{array}\right] \\
& \Gamma=P^{T} \triangle A_{0}+\triangle A_{0}^{T} P \\
& M_{2}=\left[e^{\alpha h_{1}} P^{T} \triangle A_{1}, \ldots, e^{\alpha h_{k}} P^{T} \triangle A_{k}\right]
\end{aligned}
$$

and $S$ is given by (14).

Since

$$
\begin{aligned}
S+\bar{S} & =S+\gamma_{0} F_{0} \Xi_{0}+\left(\gamma_{0} F_{0} \Xi_{0}\right)^{T} \\
& +\sum_{i=1}^{k} \gamma_{i} F_{i} \Xi_{i}+\sum_{i=1}^{k}\left(\gamma_{i} F_{i} \Xi_{i}\right)^{T}
\end{aligned}
$$

where

$$
\begin{aligned}
\gamma_{0} & =\left[D_{0}^{T} P 0 \ldots 0\right]^{T} \\
\Xi_{0} & =\left[E_{0} 0 \ldots 0\right] \\
\gamma_{i} & =\left[e^{\alpha h_{i}} D_{i}^{T} 0 \ldots 0\right] \\
\Xi_{i} & =\left[0 \ldots 0 E_{i} 0 \ldots 0\right]
\end{aligned}
$$

$E_{i}$ is the $i$-th column of $\Xi_{i}$. Using Lemma 1, we have

$$
S+\bar{S} \leq S+\sum_{i=0}^{k} \varepsilon_{i} \Xi_{i}^{T} \Xi_{i}+\sum_{i=0}^{k} \varepsilon_{i}^{-1} \gamma_{i} \gamma_{i}^{T}
$$

Using Lemma 2, we know that

$$
S+\sum_{i=0}^{k} \varepsilon_{i} \Xi_{i}^{T} \Xi_{i}+\sum_{i=0}^{k} \varepsilon_{i}^{-1} \gamma_{i} \gamma_{i}^{T}<0
$$

is equivalent to

$$
\left[\begin{array}{cccc}
X_{2} & M_{1} & K & M_{3} \\
M_{1}^{T} & -N_{2} & 0 & 0 \\
K^{T} & 0 & -\bar{N}_{1} & 0 \\
M_{3}^{T} & 0 & 0 & -N_{3}
\end{array}\right]<0
$$

where

$$
\bar{N}_{1}=\operatorname{diag}\left\{e^{-2 \alpha h_{1}} \varepsilon_{1} I_{k_{1}}, \ldots, e^{-2 \alpha h_{k}} \varepsilon_{k} I_{k_{k}}\right\}
$$

From (15) and (16), we know that (20) is true, so $S+\bar{S}<0$. Thus it is yielded that $\dot{V}<0$ whenever $\xi$ is not zero. According to the stability of the operator $D$, Lemma 3 and (13), it follows that the system (7) is robust asymptotically stable. So the system (1) is robust asymptotically stable. This completes the proof.

Remark 3 Let $k=1$, then the system (7) only has one single state delay, and (15) can be transformed into (21) as LMI problem on a single state delay.

$$
\left[\begin{array}{cccc}
\Pi & \Pi_{1} & \Pi_{2} & M_{3} \\
\Pi_{1}^{T} & -B_{1} & 0 & 0 \\
\Pi_{2}^{T} & 0 & -B_{2} & 0 \\
M_{3}^{T} & 0 & 0 & -N_{3}
\end{array}\right]<0
$$

where

$$
\begin{aligned}
\Pi & =P^{T} A_{0}+A_{0}^{T} P+\alpha P^{T} E+\alpha E P+R_{1}+\varepsilon_{0} E_{0}^{T} E \\
\Pi_{1} & =e^{\alpha h_{1}} P^{T} A_{1}, \quad \Pi_{2}=P^{T} D_{1}, \quad B_{1}=R_{1}-\varepsilon_{1} E_{1}^{T} E_{1} \\
B_{2} & =e^{-2 \alpha h} \varepsilon_{1} I_{k_{1}}, \quad M_{3}=P_{T} D_{0}, \quad N_{3}=\varepsilon_{0} I_{k_{0}}
\end{aligned}
$$

\section{Numerical Examples}

Example 1 Consider the following uncertain linear time-delay singular systems

$$
\begin{array}{r}
{\left[\begin{array}{ll}
1 & 0 \\
0 & 0
\end{array}\right]\left[\begin{array}{c}
\dot{z}_{1}(t) \\
\dot{z}_{2}(t)
\end{array}\right]=\left[\begin{array}{cc}
-1+0.1 & 0 \\
0 & -1
\end{array}\right]\left[\begin{array}{l}
z_{1}(t) \\
z_{2}(t)
\end{array}\right]} \\
+\sum_{i=1}^{2}\left[\begin{array}{cc}
-0.01 & 0 \\
0 & -0.01
\end{array}\right] e^{0.1 h_{i}} z\left(t-h_{i}\right)
\end{array}
$$

It is obvious that

$$
\begin{gathered}
\alpha=0.1, \quad E=\left[\begin{array}{ll}
1 & 0 \\
0 & 0
\end{array}\right], \quad A_{0}=\left[\begin{array}{cc}
-1 & 0 \\
0 & -1
\end{array}\right] \\
A_{i}=E=\left[\begin{array}{cc}
-0.01 & 0 \\
0 & -0.01
\end{array}\right], \quad h_{i} \leq h, i=1,2
\end{gathered}
$$

Set

$$
R_{i}=\left[\begin{array}{ll}
1 & 0 \\
0 & 1
\end{array}\right], i=1,2
$$

then

$$
N_{1}=\operatorname{diag} R_{1}, R_{2}
$$

For simple, set

$$
P=\left[\begin{array}{ll}
c & 0 \\
0 & d
\end{array}\right]
$$


where $c$ and $d$ are positive scalars and will be determined later. Noticing (11), we have

$$
\begin{aligned}
X_{1} & =\left[\begin{array}{cc}
-2 c+2 \times 0.1 c+2 & 0 \\
0 & -2 d+2
\end{array}\right], \\
M_{1} & =\left[\begin{array}{cc}
e^{0.1 h_{1}}\left[\begin{array}{cc}
-0.01 c & 0 \\
0 & -0.01 d
\end{array}\right]^{T} \\
e^{0.1 h_{2}}\left[\begin{array}{cc}
-0.01 c & 0 \\
0 & -0.01 d
\end{array}\right]^{T}
\end{array}\right]^{T}
\end{aligned}
$$

Using Lemma 2, (11) is equivalent to

$$
X_{1}+M_{1} N_{1}^{-1} M_{1}^{T}<0
$$

It is obtained that

$$
X_{1}+M_{1} N_{1}^{-1} M_{1}^{T}<\left[\begin{array}{cc}
\Sigma_{1} & 0 \\
0 & \Sigma_{2}
\end{array}\right]
$$

where

$$
\begin{aligned}
& \Sigma_{1}=-2 c+0.2 c+2+2 \times 0.01^{2} e^{0.2 h} c^{2} \\
& \Sigma_{2}=-2 d+2+2 \times 0.01^{2} e^{0} .2 h d^{2}
\end{aligned}
$$

So if

$$
\left\{\begin{array}{l}
-2 c+0.2 c+2+2 \times 0.01^{2} e^{0.2 h} c^{2}<0 \\
-2 d+2+2 \times 0.01^{2} e^{0} .2 h d^{2}<0
\end{array}\right.
$$

then

$$
X_{1}+M_{1} N_{1}^{-1} M_{1}^{T}<0
$$

Solving (23), we get

$$
\begin{aligned}
& \frac{1.8-\sqrt{\triangle_{1}}}{2 \times 2 \times 0.01^{2} e^{0.2 h}}<c<\frac{1.8+\sqrt{\triangle_{1}}}{2 \times 2 \times 0.01^{2} e^{0.2 h}} \\
& \frac{2-\sqrt{\triangle_{2}}}{2 \times 2 \times 0.01^{2} e^{0.2 h}}<c<\frac{2+\sqrt{\triangle_{2}}}{2 \times 2 \times 0.01^{2} e^{0.2 h}}
\end{aligned}
$$

where

$$
\begin{aligned}
& \triangle_{1}=1.8^{2}-4 \times 2 \times 0.01^{2} e^{0.2 h} \times 2 \\
& \triangle_{2}=2^{2}-4 \times 2 \times 0.01^{2} e^{0.2 h} \times 2
\end{aligned}
$$

Solving the following inequalities:

$$
\left\{\begin{array}{l}
\triangle_{1}>0 \\
\triangle_{2}>0
\end{array}\right.
$$

we get $h<10 \ln 45$. Hence when $h<10 \ln 45$ we have $\triangle_{1}>0, \triangle_{2}>0$. Take $c$ and $d$ satisfying (24), then the following inequality holds:

$$
X_{1}+M_{1} N_{1}^{-1} M_{1}^{T}<0
$$

According to Theorem 1, the system (22) is asymptotically stable.
Example 2 Consider the uncertain time-delay systems

$$
\begin{aligned}
& {\left[\begin{array}{ll}
1 & 0 \\
0 & 0
\end{array}\right]\left[\begin{array}{l}
\dot{z}_{1}(t) \\
\dot{z}_{2}(t)
\end{array}\right]=\left\{\left[\begin{array}{cc}
-1+0.1 & 0 \\
0 & -1
\end{array}\right]+\triangle A_{0}\right\}\left[\begin{array}{l}
z_{1}(t) \\
z_{2}(t)
\end{array}\right]} \\
& +\sum_{i=1}^{2}\left\{\left[\begin{array}{cc}
-1+0.1 & 0 \\
0 & -1
\end{array}\right]+\triangle A_{i}\right\} e^{0.1 h_{i}} z\left(t-h_{i}\right)
\end{aligned}
$$

and the uncertainties can be described by

$$
\triangle A_{j}(x, t)=D_{j} F_{j}(x, t) E_{j}, \quad j=0,1,2
$$

with

$$
D_{j}=E_{j}\left[\begin{array}{cc}
\sqrt{0.5} & 0 \\
0 & \sqrt{0.5}
\end{array}\right], F_{j}=\left[\begin{array}{cc}
\sigma_{1} & 0 \\
0 & \sigma_{2}
\end{array}\right]
$$

where $\sigma_{1}$ and $\sigma_{2}$ are unknown real parameters with $\sigma_{1}<10^{-4}, \sigma_{2}<10^{-4}$ Set

$$
R_{j}=\left[\begin{array}{ll}
1 & 0 \\
0 & 1
\end{array}\right], \quad i=1,2
$$

and

$$
P=\left[\begin{array}{ll}
c & 0 \\
0 & d
\end{array}\right]
$$

where $\mathrm{c}$ and $\mathrm{d}$ are positive scalars and will be determined later.

Noticing (11), we have

$$
\begin{aligned}
X_{1} & =\left[\begin{array}{cc}
\Sigma_{3} & 0 \\
0 & \Sigma_{4}
\end{array}\right], \\
M_{0} & =\left[\begin{array}{cc}
e^{\alpha h_{1}}\left[\begin{array}{cc}
-0.01 c+0.5 \sigma_{1} c & 0 \\
0 & -0.01 d+0.6 \sigma_{2} d
\end{array}\right]^{T} \\
e^{\alpha h_{2}}\left[\begin{array}{cc}
-0.01 c+0.5 \sigma_{1} c & 0 \\
0 & -0.01 d+0.6 \sigma_{2} d
\end{array}\right]^{T}
\end{array}\right]^{T} \\
\Sigma_{3} & =2 c\left(-1+0.5 \sigma_{1}\right)+2 \times 0.1 c+2 \\
\Sigma_{4} & =2 d\left(-1+0.6 \sigma_{2}\right)+2
\end{aligned}
$$

Using Lemma 2, (11) is equivalent to

$$
X_{0}+M_{0} N_{1}^{-1} M_{0}^{T}<0
$$

It is obtained that

$$
X_{0}+M_{0} N_{1}^{-1} M_{0}^{T}<\left[\begin{array}{cc}
\Sigma_{5} & 0 \\
0 & \Sigma_{6}
\end{array}\right]
$$

where

$$
\begin{aligned}
& \Sigma_{5}=2 c\left(-1+0.5 \sigma_{1}\right)+0.2 c+2+2\left(0.01 c-5 \sigma_{1} c\right)^{2} e^{0.2 h} \\
& \Sigma_{6}=2 d\left(-1+0.6 \sigma_{2}\right)+2+2\left(0.01 d-0.6 \sigma_{2} d\right)^{2} e^{0.2 h}
\end{aligned}
$$




$$
\begin{aligned}
& \text { Noticing } \sigma_{1}^{2}<10^{-4}, \sigma_{2}^{2}<10^{-4} \text { we get } \\
& \begin{aligned}
\Sigma_{5} & =2\left(-0.01+0.5 \sigma_{1}\right)^{2} e^{0.2 h} c^{2}+ \\
& +\left(-1.5+\sigma_{1}\right) c+2 \\
& <2 \times\left(-0.01-0.5 \times 10^{-2}\right)^{2} e^{0.2 h} c^{2} \\
& +\left(-1.8+10^{-2}\right) c+2 \\
\Sigma_{6} & =2\left(-0.01+0.6 \sigma_{2}\right)^{2} e^{0.2 h} d^{2}+ \\
& +\left(-2+1.2 \sigma_{2}\right) d+2 \\
& <2 \times\left(-0.01-0.6 \times 10^{-2}\right)^{2} e^{0.2 h} d^{2}+2 \\
& +\left(-2+1.2 \times 10^{-2}\right) d
\end{aligned}
\end{aligned}
$$

Solving the following inequalities

$$
\begin{aligned}
& 2 \times\left(-0.01-0.5 \times 10^{-2}\right)^{2} e^{0.2 h} c^{2} \\
& \quad+\left(-1.8+10^{-2}\right) c+2<0 \\
& 2 \times\left(-0.01-0.6 \times 10^{-2}\right)^{2} e^{0.2 h} d^{2}+2 \\
& \quad+\left(-2+1.2 \times 10^{-2}\right) d<0
\end{aligned}
$$

we get

$$
\begin{aligned}
& \frac{1.79-\sqrt{\triangle_{3}}}{9 \times 10^{-4} e^{0.2 h}}<c<\frac{1.79+\sqrt{\triangle_{3}}}{9 \times 10^{-4} e^{0.2 h}} \\
& \frac{1.988-\sqrt{\triangle_{4}}}{1.024 \times 10^{-3} e^{0.2 h}}<d<\frac{1.024+\sqrt{\triangle_{4}}}{1.024 \times 10^{-3} e^{0.2 h}}
\end{aligned}
$$

where

$$
\begin{gathered}
\triangle_{3}=1.79^{2}-16 \times 1.5^{2} \times 10^{-4} e^{0.2 h} \\
\triangle_{4}=1.988^{2}-16 \times 1.6^{2} \times 10^{-4} e^{0.2 h}
\end{gathered}
$$

we get $h<10 \ln \frac{179}{6}$. So when $h<10 \ln \frac{179}{6}$, we have $\triangle_{3}>0, \triangle_{4}>0$. Take $c$ and $d$ satisfy (28), then the following inequality holds:

$$
X_{0}+M_{0} N_{1}^{-1} M_{0}^{T}<0
$$

According to Theorem 1, system (25) is asymptotically stable for any

$$
\sigma_{1}^{2} \leq 10^{-4}, \quad \sigma_{2}^{2} \leq 10^{-4}
$$

\section{Conclusion}

This paper deals with the problem of robust stability criteria for a class of uncertain linear time-delay singular systems. Firstly, we consider the equivalent systems of the original systems by introducing the state transformation. The Lyapunov-Krasovskii functional is constructed, and is applied to the equivalent systems and the nominal system of the equivalent systems. The delay-dependent robust stability criterion for the equivalent systems is obtained, which also ensures the original system is asymptotically stable.

\section{Acknowledgments}

This work was supported by the Chinese National Nature Science Foundation under Grant No. 60974054.

\section{References}

[1] E. Fridman and U. Shaked, "An improved stabilization method for linear time-delay systems ", IEEE Trans. Automat. Contr., Vol. 47, No. 11, pp. 19311937, 2002.

[2] H. Gao and C. Wang, "Comments and further results on 'A descriptor system approach to control of linear time-delay systems ", IEEE Trans. Automat. Contr., Vol. 48, No. 3, pp. 520-525, 2003.

[3] J. P. Richard, "Time-delay systems: an overview of some recent advances and open problems", Automatica, Vol. 39, No. 10, pp. 1667-1694, 2003.

[4] Y. S. Lee, Y. S. Moon, W. H. Kwon, and P. G. Park, "Delay-dependent robust control for uncertain systems with a state-delay", Automatic, Vol. 40, No.1, pp. 65-72, 2004.

[5] X. Jing, D. Tan, and Y. Wang, "An LMI Approach to Stability of Systems With Severe Time-Delay", IEEE Trans. Autom. Control, Vol. 49, No. 7, pp. 11921195, 2004.

[6] C. Y. Lu, J. S. H. Tsai and T. J. Su, "On improved delay-dependent robust stability criteria for uncertain systems with multiple-state delays", IEEE Trans. on Circuits and Systems, Vol. 49, No. 2, pp. 253-256, 2002.

[7] C. Lin, Q. G. Wang, and T. H. Lee. "A less conservative robust stability test for linear uncertain timedelay systems", IEEE Trans. Automat. Contr., Vol. 51, No. 1, pp. 87-91, 2006.

[8] S. Xu, P. Van Dooren, R. Stefan, and J. Lam, "Robust stability and stabilization for singular systems with state delay and parameter uncertainty", emphIEEE Trans. Autom. Control, Vol. 47, No. 7, pp. 11221128, 2002.

[9] S. Xu and C.Yang, "An algebraic approach to the robust stability analysis and robust stabilization of uncertain singular systems", Int. J. Syst. Sci., Vol. 31, pp. 55-61, 2000. pp.43-51,2008.

[10] E. Fridman, "Stability of linear descriptor systems with delay: a Lyapunov-based approach", J. Math. Anal. Appl., Vol. 273, pp. 24-44, 2002.

[11] S. Zhu, C. Zhang, Z. Cheng, and J. Feng, "DelayDependent Robust Stability Criteria for Two Classes of Uncertain Singular Time-Delay Systems", IEEE. Trans. Autom. Control, Vol. 52, No. 5, pp. 880-885, 2007 .

[12] Y. Wang, L. Xie, and C. E. de Souza, "Robust control of a class of uncertain nonlinear systems", Syst. Contr. Lett., Vol. 19, pp. 139-149, 1992.

[13] S. Xu, J. Lam, "Robust stability and stabilization of discrete singular systems: an equivalent characterization ", IEEE Transaction on Automatic Control, Vol. 49, No. 4, pp. 568-574, 2004. 
[14] D. Yue, J. Lam, D. W. C. Ho, "Reliable control of uncertain descriptor systems with multiple delays", IEE Proceedings Control Theory \& Applications, Vol. 150, No. 6, pp. 557-564, 2003.

[15] S. Ma, Z. Cheng, C Zhang, "Delay-dependent robust stability and stabilization for uncertain discrete singular systems with time-varying delays". IET Control Theory \& Applivations, Vol. 1, No. 4, pp. 1086-1095, 2007.

[16] S. Boyd, L. El Ghaoui, E. Feron, V. Balakrishnan, "Linear Matrix Inequalities in Systems and Control Theory”, SIAM, Philadelphia, 1994.

[17] Y. He, M. Wu, J.H. She, and G.P. Liu. "Parameterdependent Lyapunov functional for stability of timedelay systems with polytopictype uncertainties". IEEE Trans. Automat. Contr., Vol. 49, No. 5, pp. 828$832,2004$. 